\title{
Contribution à l'étude photochimique de quelques plantes médicinales antidiabétiques de la ville de Bukavu et ses environs (Sud-Kivu, R.D.Congo).
}

\author{
1 MANGAMBU Mokoso Jean de Dieu, ${ }^{2}$ MUSHAGALUSA Kasali Félicien, ${ }^{3}$ KADIMA Ntokamunda Justin \\ 1 Université Officielle de Bukavu, Faculté des Sciences et Sciences Appliquées, Laboratoire de Systématique et Gestion \\ des Ressources végétales, Laboratoire de Biosystématique, BP : 570 Bukavu, R.D. Congo. Université d'Anvers/Belgique, \\ Département de Biologie, Universiteitsplein 1-C, B-2610 Antwerp-Wilrijk (Belgium). mangambu2000@yahoo.fr , \\ Mangambu@live.be et JeandeDieu.MangambuMokoso@student.ua.ac.be \\ 2 Université Officielle de Bukavu, Faculté de Médecine et Pharmacie, Département de Pharmacie, BP: 570 Bukavu, R.D. \\ Congo. felicienkasali@gmail.com et felicienkasali@yahool.fr \\ 3University of Rwanda, school of medicine and pharmacy. kntokamunda@nur.ac.rw \\ Auteur correspondant : mangambu2000@yahoo.fr et JeandeDieu.MangambuMokoso@student.ua.ac.be \\ Original submitted in on $25^{\text {th }}$ November 2013. Published online at www.m.elewa.org on $31^{\text {st }}$ March 2014. \\ http://dx.doi.org/10.4314/jab.v75i1.7
}

\section{RÉSUMÉ}

Objectif : Cette étude avait pour but de réaliser un screening phytochimique de quelques plantes médicinales utilisées pour - traiter le diabète par les tradipraticiens dans la ville de Bukavu située à l'Est de la République Démocratique du Congo (R.D.Congo).

Méthodologie et résultats : L'enquête ethnobotanique menée a permis d'identifier 30 espèces végétales utilisées contre le diabète. Parmi ces espèces 22 couramment citées par plus de la moitié des tradipraticiens ont fait l'objet de la présente étude pour détecter les grands groupes chimiques. Les échantillons comprenant feuilles, racines, écorces ont été récoltés, identifiés, séchés à l'ombre, pulvérisés, et finalement soumis au screening chimique par des techniques conventionnelles portant essentiellement sur les réactions de coloration et de précipitation. Les résultats ont montré que les composés phénoliques étaient détectés dans tous les échantillons (100\%), suivis de flavonoïdes (88,66\%) et alcaloïdes $(77,26 \%)$.

Conclusion: Cette étude est réalisée dans la perspective de mise en œuvre des initiatives innovantes pouvant déboucher dans l'avenir sur la fabrication des médicaments traditionnels pour le traitement du diabète. Elle nous a permis d'avoir une idée sur la présence des métabolites secondaires dans leurs différents extraits et ceci dans les perspectives des expériences pharmacologiques et toxicologiques futures. L'identification de certains groupes chimiques peut constituer un indicateur environnemental important quant à la conservation des espèces végétales.

Mots clés : Plantes antidiabétiques, screening phytochimique, réactions de coloration et précipitation, Bukavu, $\mathrm{RDC}$ 


\section{ABSTRACT}

Objective: This study aimed at performing a phytochemical screening of some medicinal plants used by traditional healers to treat diabetes in the city of Bukavu located in the east of the Democratic Republic of Congo (D.R.Congo).

Methodology and results: The ethnobotanical survey conducted identified 30 plant species used to treat diabetes. Of these 30 species, 22 species commonly cited by more than half of the healers were studied to detect the major chemical compounds. Samples including leaves, roots and bark, were harvested, identified, air dried in the shade, powdered, and finally submitted for chemical screening by conventional techniques focusing on colour reactions and chemical precipitation. The results showed that phenolic compounds were detected in all samples ( $100 \%)$, followed by flavonoids $(88.66 \%)$ and alkaloids $(77.26 \%)$.

Conclusion: This study showed a possibility in future of production of traditional medicines for the treatment of diabetes. It exposed the presence of secondary metabolites in their various extracts and this could lead to future pharmacological and toxicological experiments. The identification of specific chemical groups can be an important indicator of environmental conservation of plant species.

Keywords: antidiabetic plants, phytochemical screening, staining reactions and precipitation, Bukavu, D.R.Congo

\section{INTRODUCTION}

A l'heure actuelle où l'humanité fait face à des maladies de toutes sortes et où la prise en charge des questions sanitaires se révèle être un véritable problème de société, surtout dans les pays en voie de développement aux ressources insuffisantes suite à de faibles systèmes économiques (Mangambu, 2013). Un recours aux ressources locales facilement disponibles constituerait une véritable solution palliative et cela dans la perspective des objectifs du millénaire pour le développement (Kumar \& Lalramnghinglova, 2011 ; Mangambu et al., 2012). Malgré les progrès de la biologie et de la médecine de l'heure, la majorité des populations des pays en voie de développement n'ont pas accès aux soins de santé suffisants suite à de faibles systèmes économiques (Konda et al., 2011 ; Singh \& Singh, 2012 ; OMS, 2013). Pour cette raison, les ressources végétales occupent une grande place dans la vie de ces populations (Mangambu et al., 2008). Le continent africain regorge des plantes médicinales très diversifiées (Mangambu et al., 2010). Selon l'Organisation Mondiale de la Santé (OMS, 2013), plus de $80 \%$ des populations africaines font recours à la médecine et la pharmacopée traditionnelles pour faire face aux problèmes de santé (Kolling, 2010 ; Mangambu 2013). Sur plus ou moins 300.000 espèces de plantes médicinales recensées sur la planète, plus de 200.000 vivent dans les pays tropicaux de l'Afrique et ont des vertus médicinales (Kolling, 2010 ; Mangambu et al., 2012). Dans cet arsenal, les plantes utilisées pour traiter le diabète occupent une place importante. En effet, le diabète pose un problème de santé publique dans de nombreux pays car il est une cause importante de morbidité prématurée. C'est une maladie mal connue mais aussi coûteuse pour l'individu, la famille et la communauté. Les diabètes sont en fait un groupe hétérogène des maladies métaboliques dont la caractéristique principale est une hyperglycémie chronique résultant d'un défaut de la sécrétion (type -1) ou d'action d'insuline (type-2), ou de ces deux anomalies (Grimaldi, 2009 ; Kolling et al., 2010 ; Bouxid, 2012 ). Par ailleurs, le diabète peut être favorisé par les troubles de l'utilisation du glycose au niveau des cellules de tissus musculaires, les facteurs héréditaires et environnementaux ainsi que par d'autres pathologies (Deteix, 2005 ; Grimaldi, 2009 ; Bouxid, 2012). En République Démocratique du Congo $(\mathrm{RDC})$ en générale et en particulier à Bukavu le problème de santé est préoccupant, un mauvais suivie des diabétiques conduit fréquemment à la mort (Kasali et al., 2013a). La chronicité de cette affection, exige chez le diabétique un traitement à vie, bien suivi, très onéreux en milieu hospitalier, faisant appel à l'association de plusieurs thérapies 
(Bouxid, 2012) et une auto-surveillance régulière. Ces coûts prohibitifs pour les populations des pays pauvres, qui accèdent difficilement aux médicaments modernes, orientent la plupart des malades diabétiques vers les remèdes traditionnels. Souvent, les patients combinent des traitements ethnomédicaux et pharmaceutiques pour espérer obtenir rapidement la guérison (Mangambu et al., 2012 ; Singh \& Singh, 2012). Des nombreux travaux ont été réalisés sur l'activité pharmacologique des plantes antidiabétique utilisées dans la médecine traditionnelle à travers la RDC, l'Afrique et le monde. A titre illustratif, on peut citer les plantes suivantes: Allium cepa (Kook et al., 2009), Stachytarpheta indica (Silambujanaki et al., 2009), Urtica dioica (Das et al., 2011), Persea americana (Lima et al., 2012), Physalis peruvianna (Kasali et al., 2013b). Cependant, de Nos jours, force est de constater que la méconnaissance des composés chimiques responsables d'activités biologiques des plantes utilisées cause d'énormes préjudices aux prescripteurs, à la base d'irrationalisme des doses (conduisant au surdosage, au sous dosage, aux intoxications et voir même à la mort). Les doses employées restent imprécises (Bruneton, 2009). Cette imprécision constitue un véritable problème de la médecine traditionnelle. La prospection des extraits administrés de façon empirique nécessite donc une surveillance de posologie, afin d'éviter les risques réels d'accidents thérapeutiques qui peuvent parfois s'avérer tragiques (Adjoungoua et al., 2006 ; Mangambu, 2013). En plus, certaines substances chimiques présentes dans les recettes, peuvent constituer un indicateur environnemental très important quant à la conservation de ces espèces végétales (Kolling et al., 2010). Cette étude est effectuée dans la ville de Bukavu (Figure 1) qui se trouve à l'Est de la République Démocratique du Congo (RDC) située sur la rive sud-ouest du Lac Kivu.

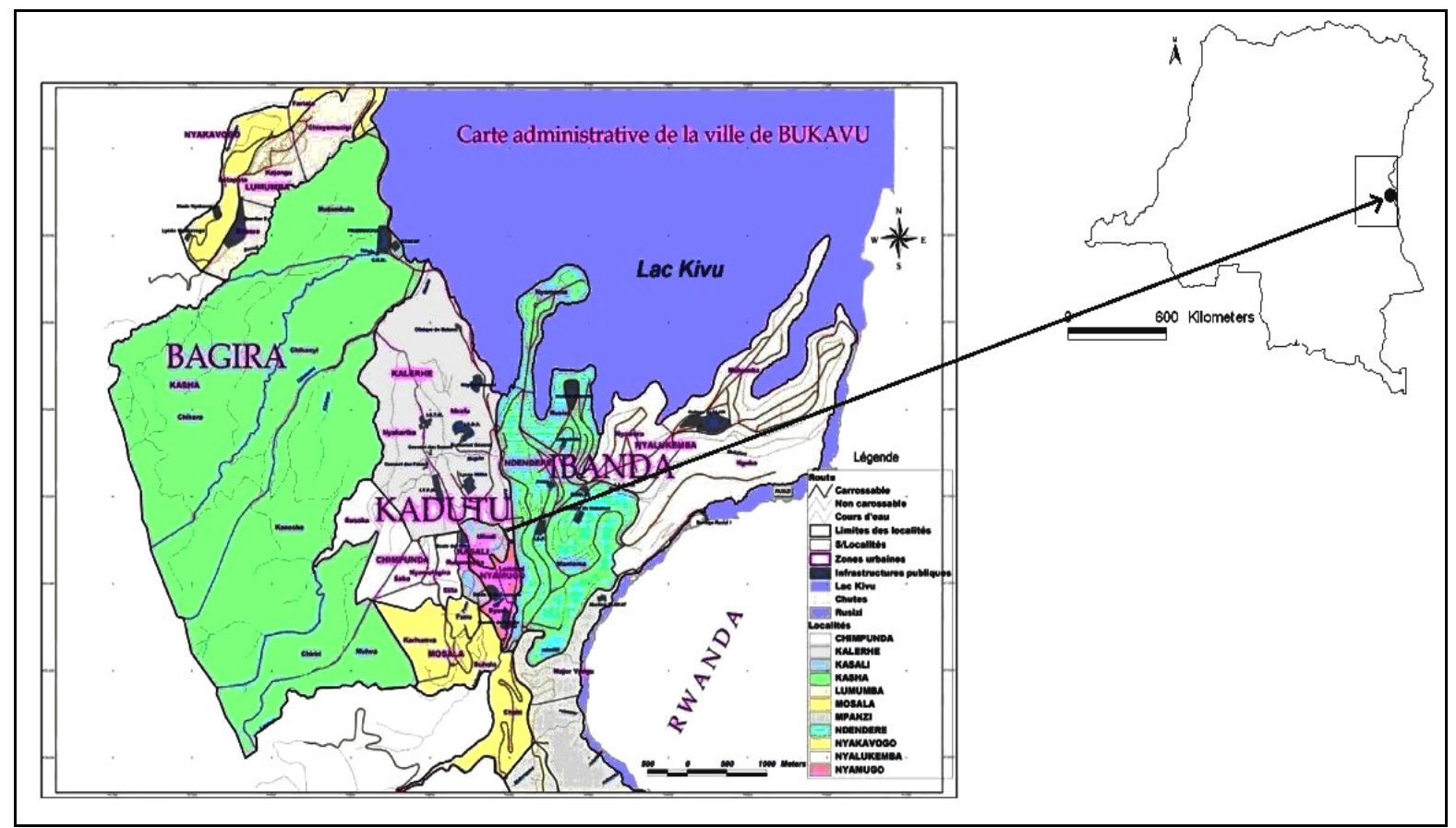

Figure 1 : Milieu d'étude. (Source : Mangambu \& Mukamba, 2007)

C'est la capitale de la province du Sud-Kivu. Elle est située à $2^{\circ} 30^{\prime}$ de latitude Sud et $28^{\circ} 5^{\prime}$ de longitude Est, à une altitude moyenne de 1600. La ville compte environ 806.940 habitants (Anonyme, 2012) et quelque 250000 autres dans la banlieue et les villages alentours (Mukamba \& Mangambu, 2007). Bien que la ville est cosmopolite dont la majorité de la population parle le Swahili, certains groupes 
ethnolinguistiques dominent, notamment les Bashi et les Lega. Elle possède un relief montagneux, et offre un climat tropical de montagne en deux saisons : une saison sèche (qui dure environ 4 mois, de mai à août) et une saison de pluie pendant les autres mois de l'année). Cette étude s'inscrit dans un programme d'amélioration et de valorisation de la médecine traditionnelle africaine par identification de quelques

\section{MATERIELS ET METHODES}

Matériel végétal : Le matériel végétal étudié est constitué des organes (feuilles, tiges feuillées, fruits, écorces, fleurs et gousses) de 22 espèces recensées dans nos inventaires. La collecte a été faite dans la ville de Bukavu et ses alentours avec le concours des tradipraticiens grâce à la collaboration de la Fédération Provinciale des Tradipraticiens de Bukavu (FPTB). La détermination et la comparaison des espèces des références ont été faites à l'Herbarium du Centre de Recherche en Sciences Naturelles de Lwiro (LWI). Les échantillons d'herbiers témoins et des photos utilisées sont déposés à LWI. Les noms des plantes ont été vérifiés suivant les systèmes basés sur l'approche phylogénétique tenant compte des données de la botanique systématique moléculaire (Angiosperm Phylogeny Group « APG » III, 2009).

Analyse au laboratoire : Les analyses ont été réalisées au Laboratoire de Pharmacognosie de la Faculté de Médecine et Pharmacie de l'Université Officielle de grands groupes chimiques ou métabolites secondaires (alcaloïdes, flavonoïdes, sucres réducteurs, quinones, polyphénols, anthocyanes, saponines, terpénoïdes, tanins). Ces métabolites sont des substances synthétisées et douées des différentes propriétés thérapeutiques exploités dans les traitements des différentes pathologies.

Bukavu. Chaque drogue fraiche récoltée a été au préalable séchée à l'ombre (Mc Calley et al., 2002). Le matériel sec a été broyé et réduit en poudre dans un mortier avant de passer au travers d'un tamis. Le screening phytochimique a été réalisé tant sur les phases aqueuses qu'organiques (Lendvai et al. 2002) par des réactions usuelles respectives des différents organes avec l'aide des réactifs de caractérisation classiques (Bruneton, 2009 ; Kolling et al., 2010). Pour chaque espèce, 50 grammes de poudre grossière ont été introduits dans un erlenmeyer contenant $100 \mathrm{ml}$ d'eau distillée ou de solvant organique (Lumbu et al., 2005). Le mélange était porté à l'ébullition pendant 30 minutes (pour l'extrait aqueux). Après filtration, les extraits bruts ainsi obtenus ont été soumis aux réactions d'identification par précipitation et par coloration suivant la méthodologie de Penge et al. (2005). Le tableau1 résume l'essentiel des procédés suivis.

Tableau 1 : tableau synthétique de groupe chimique, réaction d'identification et indicateur utilisés

\begin{tabular}{|c|c|c|}
\hline Groupe chimique & Réactifs d'identification & Indicateur \\
\hline $\begin{array}{l}\text { Alcaloïdes } \\
\text { Flavonoïdes }\end{array}$ & $\begin{array}{l}\text { Solution iodo-bismuthite de potassium } \\
\text { Acide chlorhydrique } \\
\text { Ammoniaque } \\
\text { Copaux de Zinc }\end{array}$ & $\begin{array}{l}\text { Précipité rouge-orangée } \\
\text { Coloration orange, rose violacée et rouge cerise. }\end{array}$ \\
\hline $\begin{array}{l}\text { Quinones } \\
\text { Polyphénols }\end{array}$ & $\begin{array}{l}\text { Ammoniaque concentré } \\
\text { Chlorure ferrique }(2 \%) \\
\text { Ferrocyanure de potassium (1\%) }\end{array}$ & $\begin{array}{l}\text { Coloration rouge rosâtre dans la phase aqueuse } \\
\text { Coloration bleue tendant au noir. }\end{array}$ \\
\hline $\begin{array}{l}\text { Saponosides } \\
\text { Terpénoïdes }\end{array}$ & $\begin{array}{l}\text { Indice mousse } \\
\text { Acide sulfurique concentré }\end{array}$ & $\begin{array}{l}\text { Apparition d'une mousse persistante } \\
\text { Coloration bleue }\end{array}$ \\
\hline Tanins catéchiques & $\begin{array}{l}\text { Formaldéhyde } \\
\text { Acide chlorhydrique concentré }\end{array}$ & Précipité gélatineux \\
\hline Tanins galliques & $\begin{array}{l}\text { Acétate de sodium } \\
\text { Chlorure ferrique }\end{array}$ & Coloration bleue-noire \\
\hline Sucres réducteurs & $\begin{array}{l}\text { Sulfate de Cuivre } \\
\text { Tartrate sodico-potassique }\end{array}$ & Coloration rouge brique \\
\hline Anthocyanes & Acide sulfurique à $10 \%$ & Coloration bleue \\
\hline
\end{tabular}




\section{RESULTATS ET DISCUSSION}

Ammoniaque à $50 \%$

Un total de 30 espèces de plantes antidiabétiques a été récolté pendant Nos enquêtes. Ces espèces constituent le matériel botanique de référence et sont conservées à Herbarium de Lwiro en RDC. Seulement 22 espèces utilisées par plus de la moitié des tradipraticiens sont analysées. Les résultats des tests phytochimiques sont présentés dans les tableaux 2 et 3 .

Type morphologique des espèces rencontrées: L'analyse des données morphologiques révèle que $52 \%$ des espèces sont des plantes herbacées et $48 \%$ des plantes ligneuses. Elles appartiennent à 16 familles différentes. L'utilisation prédominance des espèces herbacées serait due à la localisation géographique de la ville de Bukavu et ses environs, marquée par la présence d'une flore ligneuse qui domine (Mukamba \& Mangambu, 2007).

Tableau 2. Résultats de l'analyse phytochimique de différentes espèces végétales

\begin{tabular}{|c|c|c|c|c|c|c|c|c|c|c|c|c|}
\hline Taxons & noms communs & P.U & Alc & Flv & Qun & Phe & Sap & Ter & Tca & Tga & Sur & Ant \\
\hline \multicolumn{13}{|l|}{ ALLIACEAE } \\
\hline Allium cepa & Litungulu (SW) & $G$ & + & + & - & + & - & - & - & - & + & - \\
\hline Allium sativum & Litungulu sumu(SW) & G & + & - & - & + & + & - & + & - & - & - \\
\hline \multicolumn{13}{|l|}{ ANACARDIACEAE } \\
\hline Mangifera indica & Hembe (SW) & $F$ & - & + & - & + & - & + & - & - & + & + \\
\hline \multicolumn{13}{|l|}{ APOCYNACEAE } \\
\hline Catharanthus roseus & Vinka (SW) & $F$ & + & + & - & + & - & + & - & + & - & NT \\
\hline \multicolumn{13}{|l|}{ ASTERACEAE } \\
\hline Bidens pilosa & Nyassa (LE) & TF & - & - & - & + & + & - & + & - & + & NT \\
\hline Tithonia diversifolia & Cilula (MA) & $F$ & + & + & - & + & + & + & - & - & NT & + \\
\hline Vernonia amygdalina & Mubirizi (MA) & $F$ & + & - & - & + & - & + & - & - & + & - \\
\hline \multicolumn{13}{|l|}{ BASELLACEAE } \\
\hline Basella alba & Nderema (MA) & $F$ & - & + & - & + & + & + & + & - & - & - \\
\hline \multicolumn{13}{|l|}{ BIGNONIACEAE } \\
\hline Spathodea campanulata & Cifulula (MA) & $E$ & + & + & - & + & + & + & - & - & + & + \\
\hline \multicolumn{13}{|l|}{ CARICACEAE } \\
\hline Carica papaya & Papayi (SW) & $F$ & + & + & - & + & + & + & + & - & + & + \\
\hline \multicolumn{13}{|l|}{ FABACEAE } \\
\hline Albizia grandibracteata & Mushebeye (MA) & $E$ & + & + & - & + & + & + & + & - & - & - \\
\hline Ceasalpinia decapetala & Lurhé (MA) & $F$ & - & - & - & + & + & - & + & - & + & - \\
\hline Cassia occidentalis & Kashegema (MA) & $F$ & + & + & + & + & + & + & + & - & NT & - \\
\hline \multicolumn{13}{|l|}{ HYPERICACEAE } \\
\hline Harungana madagascariensis & Ndura (SW) & $F$ & + & + & + & + & + & + & + & - & + & - \\
\hline \multicolumn{13}{|l|}{ LAURACEAE } \\
\hline Persea americana & Ivika (MA) & $F$ & + & + & + & + & + & + & - & + & - & NT \\
\hline MALVACEAE & & & & & & & & & & & & \\
\hline
\end{tabular}




\begin{tabular}{l}
$\begin{array}{l}\text { Mangambu et al. J. Appl. Biosci. 2014. Contribution a l'étude photochimique de plantes médicinales } \\
\text { antidiabétiques de Bukavu, R.D Congo }\end{array}$ \\
\hline
\end{tabular}

\begin{tabular}{|c|c|c|c|c|c|c|c|c|c|c|c|c|}
\hline Sida acuta & Mudundu (MA) & $E$ & + & - & + & + & + & + & + & - & + & - \\
\hline \multicolumn{13}{|l|}{ PIPERACEAE } \\
\hline Piper guineense & & $\mathrm{Fr}$ & + & + & - & + & - & + & - & - & - & - \\
\hline POACEAE & Nyilulu (MA) & & & & & & & & & & & \\
\hline Zea mays & Muyindi (SW) & $S$ & - & + & + & + & - & - & - & + & + & + \\
\hline \multicolumn{13}{|l|}{ SOLANACEAE } \\
\hline Solanum americanum & Mulunda (MA) & $F$ & + & + & - & + & + & - & - & - & - & - \\
\hline Physalis peruvianna & Mbuma (MA) & $F$ & + & + & + & + & + & + & + & - & - & - \\
\hline \multicolumn{13}{|l|}{ URTICACEAE } \\
\hline Urtica dioica & Chachingi (MA) & $\mathrm{F}$ & + & + & - & + & + & + & - & - & - & + \\
\hline \multicolumn{13}{|l|}{ VERBENACEAE } \\
\hline Stachytarpheta indica & Insuline (SW ) & $F$ & + & + & + & + & + & + & - & + & NT & + \\
\hline
\end{tabular}

\section{Légende :}

Partie utilisée $(\mathrm{PU})=\mathrm{F}$ : feuille ; TF : tige feuillée ; $\mathrm{E}$ : écorce ; $\mathrm{S}$ : stigmate ; Fr : fruit ; G : Gousse

Métabolites secondaires = Alc : alcaloïdes ; Fla : flavonoïdes ; Suc réd : sucres réducteurs; Qn : quinones ; Phé : polyphénols ; Ant : anthocyanes ; Sap : saponines; Ter : terpénoïdes; Tcat : tanins catéchiques; Tgal : tanins galliques; NT : non test

Noms communs $=\mathrm{L}$ : Lega ; MA : Mashi ; SW : Swahili

Parmi les familles, les mieux représentées sont celles d'Asteraceae (Bidens pilosa, Tithonia diversifolia et Vernonia amygdalina) et Fabaceae (Albizia grandibracteata, Ceasalpinia decapetala et Cassia occidentalis) qui ont chacune trois espèces et représentent ainsi $27.27 \%$ des espèces étudiées. Les Alliaceae (Allium cepa et Allium sativum) et Solanaceae (Solanum americanum et Physalis peruvianna) représentent chacune deux espèces. Les quatre familles renferment à elles seules 10 espèces sur les 22 étudiées. Organes étudiés: Parmi les organes testés positivement, les feuilles représentent $63,63 \%$. Elles sont suivies d'écorces (13.6\%), des gousses (9.1\%) et des tiges feuillées ; le fruit et le stigmate ont chacun $4.5 \%$. L'utilisation de feuilles est justifiée par l'abondance des groupes chimiques qu'elles contiennent, car elles sont connues comme le lieu de synthèse des métabolites secondaires du végétal (Lumbu et al., 2005 ; Mangambu et al. 2008 ; Kumar \& Lalramnghinglova , 2011). Elles produisent la plupart des principes actifs notamment des alcaloïdes, essences, glucosides, tanins (Barry, 1999 ; Pamplona, 2001a).

Plantes et groupes chimiques présents : Les résultats montrent que la répartition des métabolites secondaires est inégale selon les familles, les genres et les espèces. Les tests des composés polyphénoliques se sont révélés positifs chez tous les organes considérés (100\%). La présence des flavonoïdes est marquée en tenant compte des résultats obtenus ( $86.66 \%$ ). Mais, $63,63 \%$ des tests positifs ont concerné les tanins (galliques et catéchiques). En ce qui concerne les alcaloïdes, leur test est positif chez 17 espèces (soit 77,26\%) appartenant à 11 familles différentes dont 2 appartiennent à la famille Fabaceae. Dans cette famille, nous avons trouvé les alcaloïdes dans certaines espèces (Cyrtissus scoparius, Physostignus venenosum et Galega officinalis). La présence d'alcaloïdes chez les espèces Catharanthus roseus (Apocynaceae) et Solanum americanum (Solanaceae) est justifiée par le fait que les alcaloïdes indoliques de type sécolagamine n'existent que chez les Apocynaceae, les "Loganiaceae " et les Rubiaceae (Maregesi et al.,2007 ; Kebieche, 2009 ;Kumar \& Lalramnghinglova, 2011) ; mais aussi les alcaloïdes benzylisoquinoléiques se rencontrent, entre autres, chez Persea americana, Lauraceae (Sérémé et al., 2001 ; Konda et al., 2011 ; Kumar \& Lalramnghinglova, 2011). 
Tableau 3. Pourcentage de tests positifs par organe

\begin{tabular}{lcccccccc}
\hline & Total(N) & Total(\%) & Feuille & Ecorce & Gousse & Fruit & Stigmate & Tige feuillée \\
\hline Fréquence totale & 22 & 100 & $14(63.3)$ & $3(13.6)$ & $2(9.1)$ & $1(4.5)$ & $1(4.5)$ & $1(4.5)$ \\
\hline Phénols & 22 & 100.0 & 63.6 & 13.6 & 9.1 & 4.5 & 4.5 & 4.5 \\
Flavonoïdes & 17 & 77.3 & 54.5 & 9.1 & 4.5 & 4.5 & 4.5 & 4.5 \\
Alcaloïdes & 17 & 77.3 & 50.0 & 13.6 & 9.1 & 4.5 & 0 & 0 \\
Saponines & 16 & 72.7 & 50.0 & 13.6 & 4.5 & 0 & 0 & 4.5 \\
Terpénoïdes & 16 & 72.7 & 54.5 & 13.6 & 0 & 0 & 0 & 4.5 \\
Sucre réducteur (N=19) & 10 & 52.6 & 26.3 & 10.5 & 5.3 & 0 & 5.3 & 5.3 \\
Tanins catéchiques & 10 & 45.5 & 27.3 & 13.3 & 4.5 & 0 & 4.5 & 6.7 \\
Anthocyanes (N=19) & 7 & 36.8 & 26.3 & 5.3 & 0 & 0 & 5.3 & 0 \\
Quinones & 7 & 31.8 & 22.8 & 4.5 & 0 & 0 & 4.5 & 0 \\
Tanins galliques & 4 & 18.2 & 13.6 & 0 & 0 & 0 & 4.5 & 0 \\
\hline
\end{tabular}

Nos résultats sont confirmés par d'autres recherches antérieures sur la composition phytochimique de certaines plantes (Persea americana), la présence des tanins, saponines et flavonoïdes dans les feuilles de Mangifera indica, et en plus les quinones dans celles d'Harungana madagascariensis (Kumbukana et al., 1990 ; Kumar \& Lalramnghinglova, 2011). Les analyses effectuées par Kambu (1988) sur Cassia occidentalis récoltée au Soudan, ont montré la présence des alcaloïdes, anthocyanes, hétérosides cardiotoniques, triterpènes, flavonoïdes et saponines (Kolling et al., 2010).

Activité pharmacologique des composés chimiques : La présence d'alcaloïdes, des flavonoïdes et des tanins dans les différentes espèces analysées constituent un indicateur important pour l'activité hypoglycémiante ou antidiabétique pour ces espèces végétales. II est démontré par plusieurs travaux que ces métabolites secondaires seraient doués de cette activité (Guerci et al. 2001 ; Kebieche, 2009). Les flavonoïdes sont doués des propriétés hypoglycémiantes et antidiabétiques suivant les résultats de plusieurs travaux réalisés (Guerci et al. 2001 ; Huang et al. 2004 ; Raccah, 2004 ; Punitha et al. 2006 ; Kim et al. 2006 ; Kebieche, 2009). Plusieurs mécanismes sont attribués aux flavonoïdes pour cette activité. Selon ces auteurs (Tringali 2001 ; Huang et al. 2004 ; Raccah, 2004) les flavonoïdes préviennent le diabète en inhibant l'alcalose réductase. En outre, plusieurs études ont démontré que la consommation d'aliments riches en flavonoïdes est inversement corrélée au risque de développer des maladies cardio-vasculaires (Pietta, 2000 ; Hollman, 2001). D'autre part, l'action antidiabétique des tanins est signalée par son action sur le diabète lui-même au niveau cellulaire, en favorisant l'action de l'insuline (en diminuant la résistance à l'insuline) et sur les complications du diabète par leur pouvoir antioxydant et anti enzymatique, neutralisant l'effet des radicaux libres et limitant la réaction inflammatoire dans les différents tissus (http : www.phytomania, 2010). Les alcaloïdes possèdent plusieurs applications pharmaceutiques chez l'être humain. Ces applications ont été prouvées cliniquement (Mc Calley et al. 2002 ; Lendvai B et al. 2002 ; Stöckigt J et al. 2002; Silvestrini et al. 2002). De part sa vincamine, qui est un important principe actif et un alcaloïde indolique aux propriétés vasodilatatrices notoires, la pervenche (Catharanthus roseus) contient aussi des tanins et d'autres alcaloïdes récemment identifiés. Ces alcaloïdes possèdent un discret effet hypoglycémiant, en faisant descendre le taux de glucose sanguin et réduire la glycosurie (Pamplona, 2001b). D'après d'autres études menées par Pamplona (2001b), l'action hypoglycémiante de la Morelle noire (Solanum amaricanum), serait dûe à un gluco-alcaloïde (solanine) dont la concentration est d'autant plus élevée que le climat est chaud. Nous avons aussi identifié les flavonoïdes dans les feuilles de Salvia officinalis, les glucides et autres composés dans celles de Persea americana. Dans une espèce très voisine de Salvia officinalis, en occurrence la sauge claree, les tanins, saponines, glucides, alcools aromatiques et bien d'autres, ont été reconnus. Selon une étude, l'effet hypoglycémiant significatif et durable total acétonique pourrait bien être lié à la présence à la fois des composées de types flavonoïdes et alcaloïdes agissant probablement de façon synergique (Kim et al., 2006 ; Sy et al., 2008). Cette étude est réalisée dans la perspective 
de mise en œuvre des initiatives innovantes pouvant déboucher dans l'avenir sur la fabrication des médicaments traditionnels pour le traitement du diabète en Afrique. L'identification de certains groupes chimiques peut constituer un indicateur environnemental important

\section{REMERCIEMENTS}

Nos remerciements s'adressent à l'Association des tradithérapeutes de la Ville de Bukavu, au personnel du laboratoire de pharmacognosie de l'Université Officielle

\section{RÉFÉRENCES BIBLIOGRAPHIQUES}

Adjoungoua A, Diafouka F, Koffi P, Lokrou A et Attaï H, 2006. Valorisation de la pharmacopée traditionnelle : action de l'extrait alcoolique de Bidens pilosa (Asteraceae) sur l'exploration statique et dynamique de la glycémie. Revue de Médecines et Pharmacopées Africaines, 19 : 1 12.

Anonyme, 2012. Rapport annuelle de la Marie de la ville de Bukavu.

APG III, 2009. An update of the Angiosperm Phylogeny Group classification for the orders and families of flowering plants: APG III. Botanical Journal of the Linnean Society, 161: 105-121.

Apema R, Mozouloua D, Abeye J, Salamate F. M. L. (2012). Les plantes médicinales utilisées dans le traitement du diabète par les tradipraticiens à Bangui. Pharmacopée et médecine traditionnelle africaines, $16: 121-129$

Barry MS, 1999. Les guérisseurs et leurs techniques thérapeutiques en Moyenne-Guinée. Revue de Médecines et Pharmacopées Africaines, 13 : 91-103.

Bouxid $H, 2012$. Les plantes médicinales et le diabète de type 2 (A propos de 199 cas). Thèse méd., Université Sidi Mohammed Ben Abdellah, $n^{\circ} 001 / 12$.

Bruneton J., 1999. Pharmacognosie, Phytochimie et plantes médicinales. Edition Technique et Documentation-Lavoisier, 3 e édition, Paris, 421499.

Das M, Sarma B P, Rokeya B, Parial R, Nahar N, Mosihuzzaman M, Khan A, Ali L, 2011. Antihyperglycemic and antihyperlipidemic activity of Urtica dioica on type 2 diabetic model rats. Journal of Diabetology, 2 (2): 1-6.

Grimaldi A., 2009. Traité de diabétologie, 2 ème édition, Ed. Médecine-Sciences, Flammarion, Paris, 210 p. quant à la conservation des espèces végétales. L'usage des méthodes plus perfectionnées du screening serait crucial pour compléter cette étude et l'évaluation de l'activité antidiabétique des différents extraits.

de Bukavu et aux personnes morales, pour leur assistance technique et leur soutien financier.

Guerci B, Bohme P, Kearney-Schwartz A, Zannad F et Drouin $P, 2001$. Endothelial dysfunction and type 2 diabetes. Diabetes Metab, 27: 436-447.

Hollman PCH., 2001. Evidence for health benefits of plant phenols : local or systemic effects ? Journal of the Science of Food and Agriculture 81(9), 842-852.

Huang DJ, Lin CD, Chen HJ, Lin YH, 2004. Antioxidant and antiproliferative activities of sweet potato (Ipomoea batatas [L.] Lam 'Tainong 57') constituents. Bot. Bull. Acad. Sin. 45: 179-186.

Kahungu B, Tshibangu K, Mbayo M et Lumbu S., 1999. Enquête ethnobotanique et détermination de l'activité antibactérienne de quelques plantes utilisées par des tradipraticiens de Lubumbashi (R.D.C). Anale de la Faculté de Médecine Université de Lubumbashi, 9 : 19-25.

Kasali M F., Mahano A., Bwironde F, Amani A, Mangambu JD., Nyakabwa DS., Wimba L, Tshibangu D, Ngbolua K., Kambale JK et Mpiana PT, 2013a. Ethnopharmacological survey of plants used against diabetes in Bukavu city (D.R. Congo). The Journal of Ethnobiology and Traditional Medicine. Photon, $119: 538-546$.

Kasali M F, Kadima N, Mpiana T P, Ngbolua K, Tshibangu D, 2013b. Assessment of antidiabetic activity and acute toxicity of leaf extracts from Physalis peruviana L. in guinea-pig. Asian Pacific journal of tropical Biomedicine, 3(11): $885-890$

Kebieche M, 2009. Activité biochimique des extraits flavonoïdiques de la plante Ranunculus repens L. : effet sur le diabète expérimental et l'hépatotoxicité induite par l'Epirubicine. Thèse de Doctorat en Biochimie, Université Mentouri Constantine. Faculté des Sciences de la Nature et de la Vie. 
Kim SH., Hyun SH, Choung SY, 2006. Anti-diabetic effect of cinnamon extract on blood glucose in $\mathrm{db} / \mathrm{db}$ mice. Journal of Ethnopharmacology, 104(1-2): 119-123.

Kolling M, Winkley $K$ et Von Deden M, 2010. "For someone who's rich, it's not a problem." Insights from Tanzania on diabetes health-seeking and medical pluralism among Dar es Salam's urban poor. Globalization and Health, 6:8.

Konda K, Kabukura M, Mbembe B, Itufa Y, Mahuku K, Mafuta M, Mpoyi K, Ndemankeni I, Kadima K, Kelela B, Ngiuvu V, Bongombola M et Dumu L, 2011. Plantes médicinales de traductions de la province de l'Equateur en RD CONGO). Institut de recherche en sciences de la santé (Kinshasa), $418 \mathrm{p}$.

Kook S, Kim GH, Choi K, 2009. The Antidiabetic Effect of Onion and Garlic in Experimental Diabetic Rats: Meta-Analysis. Jornal of medical food, 12:552560.

Kumar $P$ et Lalramnghinglova $H, 2011$. India with Special Reference to an Indo-Burma Hotspot Region. Ethnobotany Research \& Applications, 9: 379- 420.

Kumbukana LB, Mbuyi M, Wome B, 1990. Etat et perspectives de la recherche sur les plantes médicinales à la Faculté des Sciences de I'Université de Kisangani. Annales de la faculté des sciences de l'Université de Kisangani, 5 : 69-76.

Lendvai B, Zelles T, Rozsa B, Vizi ES, 2002. Vinca alkaloid enchanges morphological dynamics of dentric neocortical Layer 2/3 pyramidal cells. Brain Research Bulletin, 59 (4) : 257-260.

Lima CR, Vasconcelos CFB, Costa-Silva J H Maranhao C A, Costa J, Batista TM, Carneiro EM, Soares LAL, Ferreira F, Wanderley A G, 2012. Antidiabetic activity of extract from Persea americana Mill. leaf via the activation of protein kinase B (PKB/Akt) in streptozotocin-induced diabetic rats. Journal of Ethnopharmacology, 141: 517- 525.

Lumbu S., Kahumba B., Kahambwe T., Mbayo T., Kalonda M., Mwamba M., Penge O, 2005. Contribution à l'étude de quelques plantes médicinales anti diarrhéiques en usage dans la ville de Lubumbashi et ses environs, Annales de Pharmacie, 3 (1) : 75-86.

Mangambu M \& Mukamba B, 2007. Contribution à l'étude floristique des Ptéridophytes dans la ville de
Bukavu (Sud- Kivu, R.D. Congo). Rapport, Université Officielle de Bukavu, $46 \mathrm{p}$.

Mangambu M, Kamabu V, Bola MF, 2008. Les plantes médicinales utilisées dans le traitement de l'asthme à Kisangani et ses environs (Province Orientale, R.D.Congo). Annales des Sciences, Université Officielle de Bukavu, 1 (1) : 63-68.

Mangambu M., Noiha Noumi V., Zapfack L. \& Sonké B., 2010. Etude phytosociologique du groupementà piper capensis (R.D. Congo). International journal of environmental studies, 67(3) : 417-430

Mangambu M., Van Diggelen R., Mwanga Mwanga J-C., Ntahobavuka H. Malaisse F. \& Robbrecht E., 2012. Etude ethnoptéridologique, évaluation des risques d'extinction et stratégies de conservation aux alentours du Parc National de Kahuzi Biega en R.D. Congo. Geo-Eco-Trop, 36 $(1 / 2): 137-15$

Mangambu M., 2013. Taxonomie, biogéographie et écologie des Ptéridophytes de l'écosystème forestier des montagnes du Parc National de Kahuzi-Biega à l'Est de la R.D. Congo. Thèse de doctorat, Université d'Anvers/Belgique, 463 p.

Mbanya JC., Motala AA, Sobngwi E, 2010. Diabetes in sub-Saharian Africa. The Lancet, 375 : 22542266.

Maregesi SM, Ngassapa OD, Pieters L \& Vlietinck A, 2007. Ethnopharmacological survey of the Bunda district, Tanzania : Plants used to treat infectious diseases. Journal of Ethnopharmacology, 113 : 457-470.

Mc Calley DV, 2002. Analysis of the cinchona alkaloids by high-performance liquid chromatography and other separation technical. Review Journal of Chromatography, 967: 1-19.

Moswa JL, Ciamala C, Bongombola B, Nzingula N, Kapanda N, Bokatshinde O, Bunga M, 2005. Plants used for the treatment of diabetes mellitus in the Democratic Republic of Congo. Annales de Pharmacie, Vol.3 (1) : 87-93.

Muyisa KS, Bokota M et Mbuyi M, 2008. Contribution à l'analyse chimique préliminaire et à la détermination de la valeur nutritive de Cyrtosperma senegalensis (Araceae). Annales des Sciences, Université Officielle de Bukavu, 1 (1) : 114-117.

OMS. 2012. Stratégie pour la médecine traditionnelle 2007-2011. Genève 
Pamplona RG (2001). Guide des plantes médicinales. Vol.1, Bibliothèque, éducation et santé, Madrid, Paris, 232-298.

Pamplona RG (2001). Guide des plantes médicinales. Vol.2, Bibliothèque, éducation et santé, Madrid, Paris, 585-719.

Penge AO, Mwelo JN, Mbenza AP, Tshilumbu PK, Ngombe NK, Kalenda NT, Duki AM, 2005. Contribution à l'analyse chimique préliminaire et à la détermination de la valeur nutritionnelle de Cyrtosperma senegalensis (Araceae). Annales de Pharmacie, 3(1) : 153-158.

Pietta P. (2000). Flavonoids as Antioxidants. Journal of Natural Products 63(7) : 1035-1042.

Punitha ISR, Rajendran K, Shirwaikar A, Shirwaikar A, 2005. Alcoholic stem extract of Coscinium fenestratum regulates carbohydrate metabolism and improves antioxidant status in streptozotocinnicotinamide induced diabetic rats. CAM, 2(3) : 375-381.

Tringali C. (2001). Bioactive Compounds from Natural Sources: Isolation Characterization and Biological Properties. Taylor \& Francis, 36 : 339367.

Raccah D, 2004. Epidémiologie et physiopathologie des complications dégénératives du diabète sucré. EMC-Endocrinologie, 1(1) : 29-42.

Sérémé A, Millogo-Rasolodimby $\mathrm{J}$, Kouda-Bonafos $\mathrm{M}$, Guinko S, Nacro M, 2001. Vertus thérapeutiques des Anacardiaceae en liaison avec leurs métabolites et leur richesse en tanins. Annales de Botanique de l'Afrique de l'Ouest 11(1/2) : 63-71.

Silvestrini A, Pasqua G, Botta B, Monacelli B, Heijden R, Von der, Verpoorte R, 2002. Effects of alkaloid precursor feeding on a camptotheca acumisate cell line. Plant Physiol. Biochem., 40: 749-753.

Silambujanaki P, Chitra V, Soni D, Raju D, Sankari M, 2009. Hypoglycemic activity of stachytarpheta indica on streptozotocin induced wistar strain rats. International Journal of PharmTech Research, 1(4): 1564-1567.

Singh B et Singh BK, 2012. Ethnomedicinal use of Pteridophytes in reproductive health of tribal women of Pachmarhi Biosphere Reserve, Madhya Pradesh, India. International Journal of Medicine and Medical researcher, 3, 12 : 47804790.

Sofowara A, 1993. Medicinal plants and traditional medicine in Africa, 2. Spectrum Books Limited, Ibadan, Nigeria : 289.

Sofowora A, 1996. Research on Medicinal Plants and Traditional Medicine in AfricaThe Journal of Alternative and Complementary Medicine. September 2, (1/3) : 365-372

Stöckigt J, Shaludks $Y$, Unger M, Gerasimenko I, Warzecha H, Stöckigt D, 2002. Highperformance liquid chromatographic, capillary electrophoretic and capillary electrophoreticelectrospray ionization mass spectrometric analysis of selected alkaloid groups. Review Journal of Chromatography A, 967: 85-113.

Sy Gy, Faye FS, Wele A, Gueye PM, Gueye CD, Cissé A, Diaye AM, Bassene E, Faye B, 2008. Activité antihyperglycémiante de la fraction F2 de l'extrait total acétonique des feuilles de Vernonia colorata (Composeae). Pharmacopée et Médecine Traditionnelle Africaines, 15 : 6-10. 